\title{
GRB 060206: hints of precession of the central engine?
}

\author{
X. W. Liu ${ }^{1,2,3}$ X. F. Wu $u^{1,2,3,4}$, and T. Lu $u^{1,2,3}$ \\ ${ }^{1}$ Purple Mountain Observatory, Chinese Academy of Sciences, Nanjing 210008, PR China \\ e-mail: [xwliu;t.lu]@pmo.ac.cn; xfwu@caltech.edu \\ 2 National Astronomical Observatories, Chinese Academy of Sciences, Beijing 100012, PR China \\ 3 Joint Center for Particle Nuclear Physics and Cosmology of Purple Mountain Observatory - Nanjing University, Nanjing 210008, \\ PR China \\ 4 Theoretical Astrophysics 130-33, California Institute of Technology, Pasadena, California 91125, USA
}

Received 3 October 2007 / Accepted 15 May 2008

ABSTRACT

\begin{abstract}
Aims. The high-redshift $(z=4.048)$ gamma-ray burst GRB 060206 showed unusual behavior, with a significant rebrightening by a factor of $\sim 4$ at about $3000 \mathrm{~s}$ after the burst. We argue that this rebrightening implies that the central engine became active again after the main burst produced by the first ejecta, then drove another more collimated jet-like ejecta with a larger viewing angle. The two ejecta both interacted with the ambient medium, giving rise to forward shocks that propagated into the ambient medium and reverse shocks that penetrated into the ejecta. The total emission was a combination of the emissions from the reverse- and forward- shocked regions. We discuss how this combined emission accounts for the observed rebrightening.

Methods. We apply numerical models to calculate the light curves from the shocked regions, which include a forward shock originating in the first ejecta and a forward-reverse shock for the second ejecta.

Results. We find evidence that the central engine became active again $2000 \mathrm{~s}$ after the main burst. The combined emission produced by interactions of these two ejecta with the ambient medium can describe the properties of the afterglow of this burst. We argue that the rapid rise in brightness at $\sim 3000 \mathrm{~s}$ in the afterglow is due to the off-axis emission from the second ejecta. The precession of the torus or accretion disk of the central engine is a natural explanation for the departure of the second ejecta from the line of sight.
\end{abstract}

Key words. gamma rays: bursts - gamma rays: theory - gamma rays: observations

\section{Introduction}

The gamma-ray burst (GRB) 060206 at Galactic Coordinates $l=78.07 \mathrm{deg}, \quad b=78.28 \mathrm{deg}$ triggered Swift-BAT on February 6th, 04:46:53 UT (trigger time $t=0$ ) (Morris et al. 2006). It exhibited a single peak, with a duration of $T_{90}=7 \pm 2 \mathrm{~s}$ and a total fluence of $8.4 \pm 0.4 \times 10^{-7} \mathrm{erg} / \mathrm{cm}^{2}$ in the $15-$ $350 \mathrm{keV}$ band (Palmer et al. 2006). The spectroscopic redshift $z$ is 4.048 (Fynbo et al. 2006). Applying the peak energy $E_{\text {peak }}=75.4 \pm 19.5 \mathrm{keV}$, the best-fit low energy photon index $\Gamma_{1}=1.06 \pm 0.34$ and a fixed high energy photon index $\Gamma_{2}=2.5$, the isotropic-equivalent energy integrated from 1 to $10^{4} \mathrm{keV}$ in the explosion rest frame is $E_{\gamma, \text { iso }}=5.8 \times 10^{52} \mathrm{erg}$ (Palmer et al. 2006).

Swift-XRT began to observe this burst $58 \mathrm{~s}$ after the BAT trigger time. At the same time, Swift-UVOT started the on-target monitoring and detected the optical afterglow (Boyd et al. 2006). A number of ground-based telescopes performed follow up observations. The $2-\mathrm{m}$ robotic Liverpool Telescope began to observe it at $t=309 \mathrm{~s}$ and carried out multicolor $r^{\prime} i^{\prime} z^{\prime}$ photometry. In the $R$-band the light-curve exhibited three obvious bumps in the first $75 \mathrm{~min}$ including a steep rise $\left(\Delta r^{\prime} \approx-1.6\right.$ at $\left.t \approx 3000 \mathrm{~s}\right)$ (Monfardini et al. 2006). About $48.1 \mathrm{~min}$ later after the trigger time, the Rapid Telescopes for Optical Response (RAPTOR) system at Los Alamos National Laboratory began to take optical images. The obtained light curve confirmed the rebrightening from $r^{\prime} \sim 17.3$ to a peak value $r^{\prime} \sim 16.4$. The subsequent decline to $r^{\prime} \sim 16.75$ at $t=80 \mathrm{~min}$ was followed by a secondary rebrightening by $\Delta r^{\prime} \sim-0.1$ around $t=90 \mathrm{~min}$ (Woźniak et al. 2006). The MDM telescope observed a smooth break at $t_{\mathrm{b}}=0.6$ days with another bump at $t \approx 16000 \mathrm{~s}$. The overall X-ray light curve has a similar shape as the optical light curve (Stanek et al. 2007).

One of the most remarkable features of this burst is that the optical light curve had a significant rebrightening and exhibited small "bumps" and "wiggles". Similar bumps and wiggles have also been seen in a number of optical afterglows (Stanek et al. 2007). GRB 970508 had an optical afterglow light curve rather similar to that of GRB 060206 (Galama et al. 1998). The optical light curve of another recent burst, GRB 060210, also displayed a rebrightening at time $t \sim 500 \mathrm{~s}$ and a shallow decay in the early epoch. The above "unusual" behavior, which is not predicted by the standard fireball model, may be more the norm than the exception (Stanek et al. 2007).

Possible scenarios for the remarkable rebrightening in GRB 060206 at $3000 \mathrm{~s}$ are a renewed energy injection (Rees \& Mészáros 1998; Kumar \& Piran 2000; Sari \& Mészáros 2000) and a density-jump in the circum-burst medium (Dai \& Lu 2002). However, as discussed by Monfardini et al. (2006), the X-ray band frequency is above the cooling frequency at $t \sim 3000 \mathrm{~s}$, so the flux does not depend on the ambient density (Freedman \& Waxman 2001). Nakar \& Granot (2007) showed that even a sharp and large increase in the ambient medium density cannot produce a significant rebrightening as seen in the afterglow. So the rebrightening cannot be due to a density jump in the ambient medium. If the rebrightening is caused by energy injection, a huge impulsive energy injection $\Delta E \sim 1.8 E_{0}$ at $\sim 3000 \mathrm{~s}$ was required, where $E_{0}$ is the blast wave energy before the rebrightening.

In this paper, we present an alternative scenario. The central engine of this burst become active again after the initial 
burst and ejects another more collimated jet with a larger viewing angle. This jet and the initial jet sweep up the interstellar medium (ISM). The multi-wavelength emission predicted by this model can reproduce both the observed X-ray and optical data. The observational results are presented in Sect. 2. We describe the scenario in Sect. 3 and fit the remarkable optical rebrightening of GRB 060206 in Sect. 4. Finally, we summarize our results and discuss their implications in Sect. 5.

\section{Observations}

The $R$-band light curve of GRB 060206 contains five distinct bumps detected by telescopes mentioned in Sect. 1. Monfardini et al. (2006) attempted to describe the optical afterglow by applying an empirical model, which consists of a sum of smoothlyconnected broken power-law functions

$F_{v}(t)=\sum_{j} F_{j}\left[\frac{2}{\left(t / t_{j}\right)^{-\alpha_{1, j} \cdot n}+\left(t / t_{j}\right)^{-\alpha_{2, j} \cdot n}}\right]^{1 / n}$,

where $\alpha_{1, j}$ and $\alpha_{2, j}$ are the pre-bump and post-bump temporal indices of the $j$ th-bump, and $n$ is the sharpness parameter. By fitting the $R$-band light curve, they are able to obtain temporal information about every bump and the break at $53000 \mathrm{~s}$ of postbreak index $\alpha=1.79 \pm 0.11$ (see Table 2 of Monfardini et al. 2006). The post-bump indices of the 1 st, 2 nd, and 4 th bumps are $\alpha_{2}=1.2 \pm 0.5, \sim 1.0$ and $0.95 \pm 0.02$, respectively, which are consistent with a typical value $3(p-1) / 4$ or $(3 p-2) / 4$ in the normal decay phase predicted by the standard afterglow model (Sari et al. 1998). If we fit the 5th bump independently, its post-bump index follows obviously the normal decay slope. As shown in Stanek et al. (2007), the X-ray light curve demonstrated similar behavior to the optical light curve. There were only two X-ray observations before $\sim 3000 \mathrm{~s}$ of temporal index $\alpha=1$. The later $\mathrm{X}$-ray light curve exhibited clear short timescale variations, but corresponding bumps were not seen.

Multi-epoch spectral energy distribution (SED) analysis revealed either an SED evolution or an additional unresolved activity (or both) during the early time interval from $t \sim 1000 \mathrm{~s}$ to $t \sim 3000 \mathrm{~s}$ based on the $i^{\prime}$ and $z^{\prime}$ photometric data (Monfardini et al. 2006). At a later time, the infrared to X-ray fluxes (after a significant rebrightening) can be fitted by a single power law with a spectral index $\beta=0.93 \pm 0.02$. However, a broken power law with $\beta_{\mathrm{OPT}}=0.7$ and $\beta_{\mathrm{X}}=1.2$ cannot be ruled out.

\section{Scenario}

It is generally accepted that long GRBs (duration $>2$ s) originate in collapsars (Woosley 1993; MacFadyen \& Woosley 1999), while short GRBs, of duration less than $2 \mathrm{~s}$, are associated with the merging of compact objects (see Nakar 2007, for a review). In both scenarios of GRB origins, a hot and dense accretion disk formed possibly around the GRB central engine. Reynoso et al. (2006) argued that a rotating black hole could induce the surrounding neutrino-cooled accretion disk to precess and nutate. The precession period varied between approximately $0.01 \mathrm{~s}$ and $10 \mathrm{~s}$ for typical central engines. Therefore, even if the jet producing the main burst is along the line-of-sight (LOS), the collimated outflow supplied by the late activity of the central engine is possibly off-LOS due to the precession of either the torus or the accretion disk. The interaction of the off-LOS outflow with the ambient medium could play an important role in the GRB afterglow emissions, e.g., produce a rebrightening as observed in the afterglow of GRB 060206.

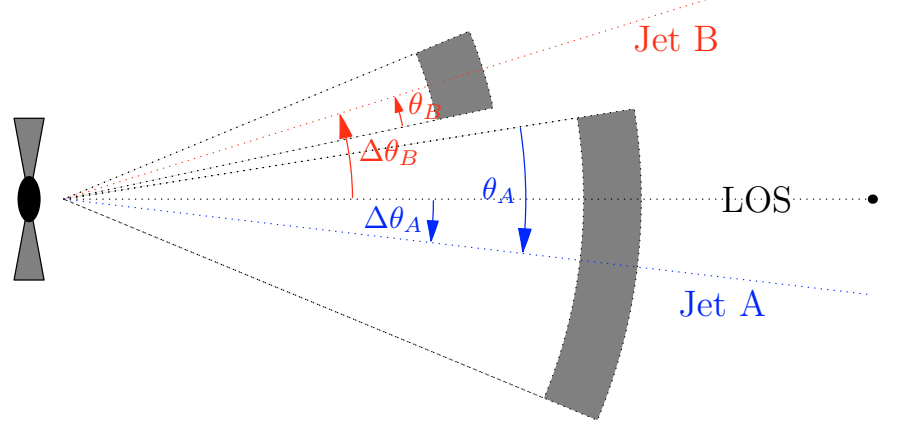

Fig. 1. Schematic two jets scenario for GRB 060206.

In our scenario illustrated in Fig. 1, the accretion-powered GRB central engine firstly generates a jet (denoted by Jet $A$ ) along LOS, which produces the observed prompt $\gamma$-ray emission. The interaction between Jet $A$ and the ambient medium is responsible for the first part of the afterglow until the large rebrightening. A period $\Delta t$ later, the central engine ejects the second jet (denoted by Jet $B$ ) at a larger viewing angle preventing $\gamma$-ray detection. By assuming that the isotropic energy of Jet $B$ is, however, significantly higher than that of Jet $A$, the off-axis afterglow emission from Jet $B$ produces significant rebrightening at lower energies. The two jets should also not intersect, and therefore collide, with each other.

Pre-large rebrightening. Emission from the forward shock, driven by Jet $A$, interacts with the ambient medium to produce an afterglow for up to $3000 \mathrm{~s}$; during this time, the temporal decay indices of the two small bumps are similar to the values predicted by the standard fireball model. There is no signature of reverse shock emission, which possibly ceases at very early times. Alternatively, the reverse shock emission could be suppressed by the magnetization of the ejecta (Zhang \& Kobayashi 2005) and many other physical processes (Kobayashi 2000; Nakar \& Piran 2004; Kobayashi et al. 2007; Jin et al. 2007). We consider that the cold Jet $A$ with a total isotropic kinetic energy $E_{\text {iso }}^{A}=10^{52} E_{52}$ erg propagates into the ambient medium with a constant density $n_{1}$. A forward shock emerges and energizes the surrounding materials by converting the bulk kinetic energy of the jet into the internal energy of the shocked materials. This internal energy is assumed to be shared by electrons and magnetic fields with energy equipartition factors $\epsilon_{\mathrm{e}}^{A}$ and $\epsilon_{B}^{A}$, respectively. If the shock is adiabatic, the synchrotron emission produced by slow cooling electrons is expected to have a peak flux

$$
F_{v, \max }=1.1 \times 10^{5} \epsilon_{B}^{A^{1 / 2}} E_{52} n_{1}^{1 / 2} D_{28}^{-2}(1+z) \mu \mathrm{Jy}
$$

at time

$$
t_{\mathrm{m}}=0.69 \epsilon_{B}^{A^{1 / 3}} \epsilon_{\mathrm{e}}^{A^{4 / 3}} E_{52}^{1 / 3} v_{15}^{-2 / 3}(1+z)^{1 / 3} \text { day }
$$

which is defined to be the time at which the typical synchrotron frequency $v_{\mathrm{m}}$ crosses the observed frequency $v_{\mathrm{obs}}=10^{15} v_{15} \mathrm{~Hz}$ (Sari et al. 1998). The luminosity distance of the burst is $D_{\mathrm{L}}=10^{28} D_{28} \mathrm{~cm}^{2}$.

For GRB 060206, no early flux peaks were detected in the optical and X-ray bands, even at $t \sim 200 \mathrm{~s}$. It is reasonable to believe that the flux peak time was less than $\sim 0.002$ day. A lower limit to the peak flux $F_{v, \max } \sim 1000 \mu \mathrm{Jy}$ can be obtained accordingly, by extrapolating the $R$-band flux of the first post-bump back to $200 \mathrm{~s}$. Since the optical flux peak was at time $t_{\mathrm{m}}$ and a cooling break in the X-ray afterglow was not observed, the order 
of the frequencies, after the flux peak, was $v_{\mathrm{m}}<v_{\mathrm{obs}}<v_{\mathrm{c}}<v_{\mathrm{X}}$, where $v_{\mathrm{c}}$ is the cooling frequency and $v_{\mathrm{X}}$ is the typical $\mathrm{X}$ ray frequency. The redshift of GRB 060206 is known, so the isotropic energy of its first jet $E_{\text {iso }}^{A}$ is $\sim 5.8 \times 10^{52} \mathrm{erg}$. The energy equipartition factors of magnetic fields and electrons, according to Eqs. (2) and (3), can be constrained if the number density of the ambient medium is known. The spectral analysis revealed a high number density in the environment of GRB 060206 (Fynbo et al. 2006). Given a rational value of $n_{1}=50 \mathrm{~cm}^{-3}$, it can be proven that $\epsilon_{B}^{A} \geq 3 \times 10^{-5}$ and $\epsilon_{\mathrm{e}}^{A} \leq 0.05$. Our subsequent numerical results are consistent with this constraint. We calculate the forward shock synchrotron emission from Jet $A$ that reproduces the first post-bump segment of the $R$-band light curve of temporal index $\alpha_{2}=1.2 \pm 0.5$, which implies an electron energy spectral index $p=4 \alpha_{2} / 3+1=2.6 \pm 1.6$.

Large rebrightening. The large rebrightening is due to the off-axis emission from the second beamed jet. The off-axis effects can generate a fast rise light curve, and the gamma-ray emission, from internal shocks in the second jet, would not trigger Swift-BAT due to the large viewing angle and the initially large Lorentz factor when the condition $\Delta \theta_{B}-\theta_{B}>1 / \Gamma_{B}$ is fulfilled, where $\Gamma_{B}$ is the initial bulk Lorentz factor of Jet B. The half opening angle of Jet B can be estimated by the measured jet break time $t_{\mathrm{b}}=53000 \mathrm{~s}$ (e.g., Frail et al. 2001)

$$
\begin{aligned}
\theta_{B} \sim & 0.057\left(\frac{t_{\mathrm{b}}}{1 \text { day }}\right)^{3 / 8}\left(\frac{1+z}{2}\right)^{-3 / 8}\left(\frac{E_{\text {iso }}^{B}}{5 \times 10^{53} \mathrm{erg}}\right)^{-1 / 8} \\
& \times\left(\frac{n_{1}}{0.1 \mathrm{~cm}^{-3}}\right)^{1 / 8} .
\end{aligned}
$$

The half opening angle and isotropic kinetic energy $E_{\text {iso }}^{B}$ of Jet $B$ can be obtained only by numerical fitting to the rebrightening and the late afterglow light curve.

\section{Numerical method}

When Jet $A / B$ sweeps up the ambient medium, a pair of shocks could be generated, including a forward shock propagating into the medium and a reverse shock penetrating into the ejecta. However, as analyzed above, the reverse shock driven by Jet $A$ can be ignored for the afterglow phase of interest, whereas it may be necessary to consider the contribution of the reverse shock to the afterglow emissions for Jet $B$. We would therefore describe the dynamics of the two jets in different ways.

\subsection{Dynamics}

\subsubsection{Jet $A$}

The evolution of the forward shock driven by Jet $A$ with an initial Lorentz factor $\Gamma_{A}$ follows the equation (Huang et al. 2000)

$\frac{\mathrm{d} \gamma_{2}^{A}}{\mathrm{~d} R_{A}}=-2 \pi\left(1-\cos \theta_{A}\right) R_{A}{ }^{2} n_{1} m_{\mathrm{p}} \frac{\gamma_{2}^{A^{2}}-1}{\varepsilon_{2}^{A} m_{2}^{A}+2\left(1-\varepsilon_{2}^{A}\right) \gamma_{2}^{A} m_{2}^{A}}$,

where $m_{\mathrm{p}}$ is the mass of the proton, $\gamma_{2}^{A}$ is the bulk Lorentz factor of the forward shock, and $\varepsilon_{2}^{A}$ is the radiative efficiency of the forward-shocked electrons. In our calculations, we take adiabatic shock assumption and thus $\varepsilon_{2}^{A}=0$. The swept-up mass $m_{2}^{A}$ by the shock is determined by

$\frac{\mathrm{d} m_{2}^{A}}{\mathrm{~d} R_{A}}=2 \pi R_{A}^{2}\left(1-\cos \theta_{A}\right) n_{1} m_{\mathrm{p}}$
Finally, for describing the temporal behavior of the shock, we refer to the equation

$$
\frac{\mathrm{d} R_{A}}{\mathrm{~d} t}=\frac{\beta_{2}^{A}}{1-\beta_{2}^{A}} \frac{c}{1+z} .
$$

\subsubsection{Jet $B$}

With respect to Jet $A$, the consideration of Jet $B$ is more complicated because of the involvement of the reverse shock. The system is divided into four regions by the two shocks and the contact discontinuity surface: the unshocked medium, the shocked medium, the shocked ejecta, and the unshocked ejecta, which is denoted by $1-4$, respectively. Since the Lorentz factor $\gamma$ and energy density $e$ are continuous along the contact discontinuity, we have $\gamma_{2}^{B}=\gamma_{3}^{B}$ and $e_{2}^{B}=e_{3}^{B}$ (Sari \& Piran 1995). The hydrodynamics of the forward-reverse shock pairs are determined by (Huang et al. 2000; Yan et al. 2007)

$\frac{\mathrm{d} \gamma_{2}^{B}}{\mathrm{~d} R_{B}}=-2 \pi R_{B}^{2}\left(1-\cos \theta_{B}\right) \frac{Q}{P}$

where the convenient parameters $Q$ and $P$ are defined in Yan et al. (2007) to be

$$
\begin{aligned}
Q= & \left(\gamma_{2}^{B^{2}}-1\right) n_{1} m_{\mathrm{p}}+\left(\gamma_{2}^{B} \gamma_{34}^{B}-\gamma_{4}^{B}\right)\left(\gamma_{4}^{B} n_{4} m_{\mathrm{p}}\right)\left(\beta_{4}^{B}-\beta_{R S}^{B}\right) \\
P= & m_{2}^{B}+m_{3}^{B}+\left(1-\varepsilon_{2}^{B}\right)\left(2 \gamma_{2}^{B}-1\right) m_{2}^{B}+\left(1-\varepsilon_{3}^{B}\right)\left(\gamma_{34}^{B}-1\right) m_{3}^{B} \\
& +\left(1-\varepsilon_{3}^{B}\right) \gamma_{2}^{B} m_{3}^{B}\left(\gamma_{4}^{B}-\frac{\gamma_{4}^{B} \beta_{4}^{B}}{\beta_{2}^{B}}\right)
\end{aligned}
$$

where $\beta_{2}^{B}, \beta_{4}^{B}$ and

$\beta_{\mathrm{RS}}^{B}=\frac{\gamma_{3}^{B} \beta_{3}^{B} n_{3}^{B}-\gamma_{4}^{B} \beta_{4}^{B} n_{4}^{B}}{\gamma_{3}^{B} n_{3}^{B}-\gamma_{4}^{B} n_{4}^{B}}$

are the velocities of the shocked medium, unshocked ejecta, and the reverse shock in the observer's frame, respectively. The relative Lorentz factor of region 3 with respect to region 4 is $\gamma_{34}^{B}$. The radiative efficiency $\varepsilon_{i}^{B}$ of region $i(i=2,3)$ is equal to zero for the adiabatic shock. The mass $m_{2}^{B}$ swept up by the forward shock and $m_{3}^{B}$ by the reverse shock, can be calculated respectively, by

$\frac{\mathrm{d} m_{2}^{B}}{\mathrm{~d} R_{B}}=2 \pi R_{B}^{2}\left(1-\cos \theta_{B}\right) n_{1} m_{\mathrm{p}}$

$\frac{\mathrm{d} m_{3}^{B}}{\mathrm{~d} R_{B}}=2 \pi R_{B}^{2}\left(1-\cos \theta_{B}\right)\left(\beta_{4}^{B}-\beta_{\mathrm{RS}}^{B}\right) \gamma_{4}^{B} n_{4}^{B} m_{\mathrm{p}}$,

where the comoving number density of the unshocked ejecta is expressed as $n_{4}^{B}=E_{\text {iso }}^{B} /\left(4 \pi R_{B}{ }^{2} \gamma_{4}^{B} \Delta m_{\mathrm{p}} c^{2}\right)$ where $E_{\text {iso }}^{B}$ and $\Delta$ are the kinetic energy and the width of Jet $B$, respectively. The temporal evolution of the radius $R_{B}$ of Jet $B$ satisfies the same equation as that of Jet $A$, which reads

$\frac{\mathrm{d} R_{B}}{\mathrm{~d} t}=\frac{\beta_{2}^{B}}{1-\beta_{2}^{B}} \frac{c}{1+z}$

After the reverse shock crosses the ejecta, the Lorentz factor of the reverse-shocked ejecta follows $\gamma_{3}^{B} \propto R_{B}{ }^{-7 / 2}$ and the mass of the shocked ejecta remains constant $m_{3}^{B}=M_{\mathrm{ej}}^{B}=E_{\mathrm{iso}}^{B} /\left(\Gamma_{B} c^{2}\right)$. 
However, the forward shock continues to sweep up the external medium, evolving in a similar way to Jet $A$ described by Eq. (5) as

$\frac{\mathrm{d} \gamma_{2}^{B}}{\mathrm{~d} R_{B}}=-2 \pi\left(1-\cos \theta_{B}\right) R_{B}{ }^{2} n_{1} m_{\mathrm{p}} \frac{\gamma_{2}^{B^{2}}-1}{\varepsilon_{2}^{B} m_{2}^{B}+2\left(1-\varepsilon_{2}^{B}\right) \gamma_{2}^{B} m_{2}^{B}}$.

\subsection{Synchrotron emission}

Due to the existence of the shocks, the bulk kinetic energy of the ejecta should be gradually converted to the internal energy $e$ of the shocked materials, which is shared between magnetic fields, electrons and protons according to the fractions $\epsilon_{B}, \epsilon_{\mathrm{e}}$ and $1-$ $\epsilon_{\mathrm{e}}-\epsilon_{B}$, respectively.

It is accepted in general that the synchrotron radiation of the shocked electrons produces the observed X-ray and optical afterglow emissions. In a detailed calculation, we consider, as often assumed, that the electrons without energy losses are accelerated by the shocks in a way described by a power law distribution

$\frac{\mathrm{d} N_{\mathrm{e}}}{\mathrm{d} \gamma_{\mathrm{e}}} \propto \gamma_{\mathrm{e}}^{-\mathrm{p}}, \quad \gamma_{\mathrm{m}}<\gamma_{\mathrm{e}}<\gamma_{\mathrm{M}}$

with a minimum Lorentz factor $\gamma_{\mathrm{m}}$ and a maximum Lorentz factor $\gamma_{\mathrm{M}}$. Another critical Lorentz factor $\gamma_{\mathrm{c}}$, above which the energy losses of the electrons due to both synchrotron and inverse Compton (IC) radiation is significant, was derived by Sari et al. (1998). The actual distribution of the electrons should be given according to the following cases (Sari et al. 1998; Yan et al. 2007) as

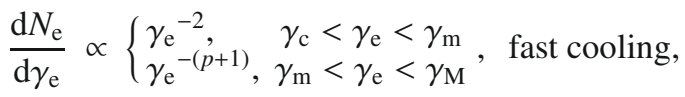

$\frac{\mathrm{d} N_{\mathrm{e}}}{\mathrm{d} \gamma_{\mathrm{e}}} \propto\left\{\begin{array}{ll}\gamma_{\mathrm{e}}^{-p}, & \gamma_{\mathrm{m}}<\gamma_{\mathrm{e}}<\gamma_{\mathrm{c}} \\ \gamma_{\mathrm{e}} & \end{array}\right.$, slow cooling.

To be specific, the three characteristic Lorentz factors for the distribution of the electrons can be calculated by $\gamma_{\mathrm{m}} \approx$ $\epsilon_{\mathrm{e}}\left(m_{\mathrm{p}} / m_{\mathrm{e}}\right)(p-2) /(p-1) \gamma_{\mathrm{rel}}, \gamma_{\mathrm{M}} \approx 10^{8}(B / 1 \mathrm{G})^{-1 / 2} /(1+Y)$ and $\gamma_{\mathrm{c}}=6 \pi m_{\mathrm{e}} c(1+z) /\left[(1+Y) \sigma_{T} \gamma B^{2} t\right]$, where the magnetic field strength $B=\sqrt{8 \pi \epsilon_{B} e}$, the relative Lorentz factor $\gamma_{\mathrm{rel}}$ is taken to be $\gamma_{34}$ for the reverse-shocked region and $\gamma_{2}$ for the forwardshocked region, and the Compton parameter $Y$ is defined as the ratio between the IC and synchrotron luminosities (Sari et al. 1998; Sari \& Esin 2001).

Using the derived electron distribution, we can calculate the synchrotron emissivity to be (Rybicki \& Lightman 1979)

$\varepsilon^{\prime}\left(v^{\prime}\right)=\frac{\sqrt{3} q_{\mathrm{e}}^{3} B}{m_{\mathrm{e}} c^{2}} \int \mathrm{d} \gamma_{\mathrm{e}} \frac{\mathrm{d} N_{\mathrm{e}}}{\mathrm{d} \gamma_{\mathrm{e}}} f\left(\frac{v^{\prime}}{v_{*}}\right)$,

where $q_{\mathrm{e}}$ is the electron charge, $v_{*}=3 \gamma_{\mathrm{e}}^{2} q_{\mathrm{e}} B /\left(4 \pi m_{\mathrm{e}} c\right), f(x)=$ $x \int_{x}^{\infty} K_{5 / 3}(k) \mathrm{d} k$ with $K_{5 / 3}(k)$ being the modified Bessel function. By integrating over all of the emitting regions, we derive the observed synchrotron flux density at a frequency $v$ to be (Huang et al. 2000)

$F_{\gamma}(t)=\frac{1}{4 \pi D_{L}^{2}} \int \mathrm{d} V^{\prime} \frac{\varepsilon^{\prime}[\gamma(1-\beta \mu) \nu(1+z)]}{[\gamma(1-\beta \mu)]^{3}}$,

where $\mu=\cos \Theta$ is the cosine value of the angle $\Theta$ between the velocity of emitting materials and LOS. Taking into account the time delay between the emissions from different latitudes, the above integration should be performed on the so-called equal-arrival-time surface that is determined by (Huang et al. 2000)

$t=\int \frac{1-\beta \mu}{\beta c} \mathrm{~d} R \equiv$ const.

From the obtained numerical synchrotron spectra, two break frequencies appear, that is, $v_{\mathrm{m}} \simeq \gamma \gamma_{\mathrm{m}}{ }^{2} q_{\mathrm{e}} B / 2 \pi m_{\mathrm{e}} c$ and $v_{\mathrm{c}} \simeq$ $\gamma \gamma_{\mathrm{c}}{ }^{2} q_{\mathrm{e}} B / 2 \pi m_{\mathrm{e}} c$, which correspond to the characteristic Lorentz factors $\gamma_{\mathrm{m}}$ and $\gamma_{\mathrm{c}}$, respectively. The peak flux at $\min \left\{v_{\mathrm{m}}, v_{\mathrm{c}}\right\}$ is $F_{\max }=\sqrt{3} \Phi q_{\mathrm{e}}^{3} B N_{\text {tot }} / m_{\mathrm{e}} c^{2}$, where $\Phi$ is an integrating coefficient from Eq. (18) (Wijers \& Galama 1999). These characteristic quantities are applied in our analysis in Sect. 3.

We also take into account the synchrotron self-absorption effect, which implies that a correction should be applied to spectra below the synchrotron self-absorption frequency $v_{\mathrm{a}}$ as performed by Wu et al. (2003) and Zou et al. (2005).

\subsection{Fitting the afterglow data}

Using the model described above with parameter values as listed in Table 1 , we describe numerically the $R$-band afterglow data of GRB 060206 in Fig. 2. As can be seen, the first post-bump in the light curve can be reproduced well using the forward shock emission from Jet $A$. Both the forward- and reverse-shock light curves of Jet $B$ are also presented in Fig. 2. The large rebrightening can be attributed mainly to the forward shock emission from Jet $B$ because the off-axis effect suppresses the peak flux of the reverse-shock emission, which usually peaks at a few hundred seconds from its beginning. For Jet $A$, we observe that the values of $\epsilon_{\mathrm{e}}^{A}, \epsilon_{B}^{A}$, and $n_{1}$ are consistent with our analysis in Sect. 3. For Jet $B$, we adopt typical values of 0.1 for $\epsilon_{\mathrm{e}}^{B}$ and 0.01 for $\epsilon_{B}^{B}$ since there is no observational constraint on the shock parameters. The shock parameters $\left(\epsilon_{\mathrm{e}}, \epsilon_{B}, p\right)$ for different jets and/or for different shocked regions may be different as found for the twocomponent jets model (Jin et al. 2007) and the forward-reverse shock model (Fan et al. 2002). The off-axis effect alone cannot explain the fast rise in the large rebrightening. In addition, the zero time effect can steepen the rise further (Zhang et al. 2006; Liang et al. 2006). We find that the time delay between the two jets is $\Delta t=2000 \mathrm{~s}$, which agrees with the rising segment, the subsequent normal decay phase, and the break in the light curve at late times.

It should be noted that the parameters $\left(\theta_{A}, \Delta \theta_{A}, \Gamma_{A}\right)$ for Jet $A$, $\left(\theta_{B}, \Delta \theta_{B}, \epsilon_{\mathrm{e}}^{B}, \epsilon_{B}^{B}, \Gamma_{B}, E_{\text {iso }}^{B}, \Delta\right)$ for Jet $B$ and the number density of the ambient medium $n_{1}$ are not exclusively determined. The jet break time for Jet $A$ is hidden by the dominating emission of Jet $B$, while the isotropic kinetic energy of Jet $B$ cannot be measured because its gamma-ray emission cannot trigger the detector. The most appropriate method for determining the parameter values depends upon the following ingredients: (1) we fit the observational $R$-band data of the first post-bump segment to constrain the values of $\theta_{A}$ and $\Gamma_{A}$ combined with the known isotropic gamma-ray energy of Jet $A$, (2) typical values of $\epsilon_{\mathrm{e}}^{B}$, and $\epsilon_{B}^{B}$ are used for Jet $B,(3)$ the off-axis angle $\Delta \theta_{B}$ cannot be too large, otherwise an abnormally large isotropic energy for Jet $B$ is required, (4) the condition of $\Delta \theta_{B}-\theta_{B}>1 / \Gamma_{B}$ should be satisfied to avoid the detection of gamma-ray emission from Jet $B$, and (5) to simplify the calculation, we assume that the path of Jet $A$ does not intersect with that of Jet $B$, which requires that $\theta_{A}-\Delta \theta_{A} \leq \Delta \theta_{B}-\theta_{B}$. 
Table 1. The main parameters adopted in our calculations.

\begin{tabular}{|c|c|c|c|c|}
\hline & & Jet $A$ & \multicolumn{2}{|c|}{ Jet $B$} \\
\hline Symbols & Definitions of the Symbols & Forward Shock & Forward Shock & Reverse Shock \\
\hline$E_{\text {iso }}^{l}(\mathrm{erg})$ & Isotropic Energy & $5.8 \times 10^{52}$ & \multicolumn{2}{|c|}{$1.0 \times 10^{54}$} \\
\hline$\Gamma_{l}$ & Initial Lorentz Factor & 300 & \multicolumn{2}{|c|}{300} \\
\hline$n_{1}\left(\mathrm{~cm}^{-3}\right)$ & Number Density of ISM & 50.0 & \multicolumn{2}{|c|}{50.0} \\
\hline$p_{l}$ & Electron Spectral Index & 2.10 & \multicolumn{2}{|c|}{2.12} \\
\hline$\theta_{l}$ & Half Opening Angle & 0.08 & \multicolumn{2}{|c|}{0.04} \\
\hline$\Delta \theta_{l}$ & Viewing Angle & 0.07 & \multicolumn{2}{|c|}{0.05} \\
\hline$\varepsilon_{i}^{l}$ & Radiative Efficiency & 0.0 & 0.0 & 0.0 \\
\hline$\epsilon_{\mathrm{e}, i}^{l}$ & Electron Equipartition Factor & 0.05 & 0.1 & 0.1 \\
\hline$\epsilon_{B, i}^{l, l}$ & Magnetic Equipartition Factor & 0.0008 & 0.01 & 0.01 \\
\hline & \multicolumn{4}{|c|}{ Values Of Following Physical Quantities At $t \sim 3000 \mathrm{~s}$} \\
\hline$\gamma_{i}^{l}$ & Bulk Lorentz Factor & 22 & 29 & 32 \\
\hline$\gamma_{\mathrm{m}, i}^{l}$ & Minimal Lorentz Factor & 180 & 540 & 3.5 \\
\hline$\gamma_{\mathrm{c}, i}^{l}$, & Cooling Lorentz Factor & 2600 & 205 & 3.5 \\
\hline$\gamma_{\mathrm{M}, i}^{l, l}$ & Maximal Lorentz Factor & $7.6 \times 10^{7}$ & $3.6 \times 10^{7}$ & $4.8 \times 10^{7}$ \\
\hline$B_{i}^{l}(\mathrm{G})$ & Comoving Magnetic Field Strength & 1.7 & 7.8 & 4.3 \\
\hline$m_{i}^{l}(\mathrm{~g})$ & Shocked Mass & $1.2 \times 10^{29}$ & $5.2 \times 10^{29}$ & $3.7 \times 10^{30}$ \\
\hline
\end{tabular}

Notes: for Jet $A, l=A$ and for Jet $B, l=B$. The subscript $i=2$ represents forward-shocked region and $i=3$ represents reverse-shocked region. In the lower part of the table, some physical quantities involved in the calculations at $t \sim 3000 \mathrm{~s}$ are presented for illustration.

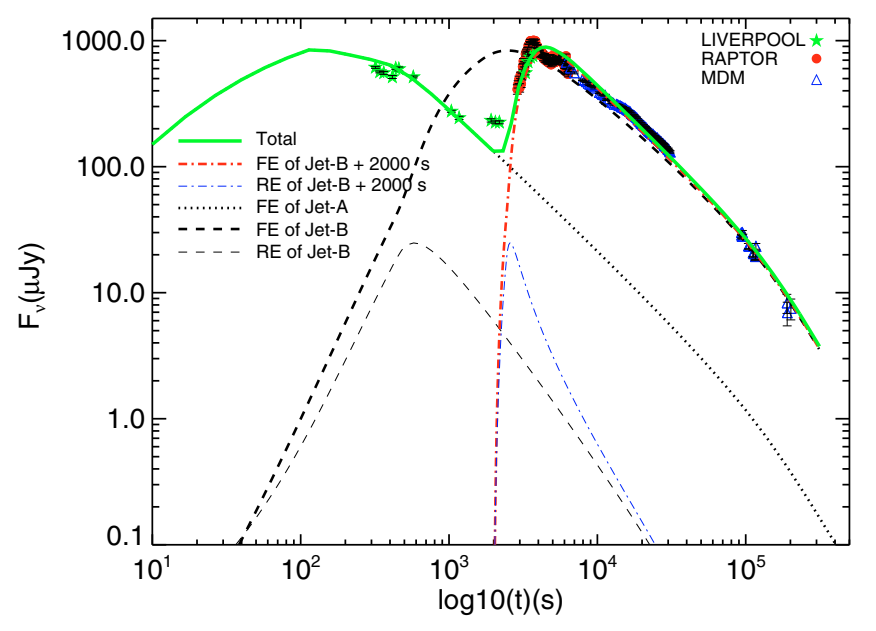

Fig. 2. $R$-band light curve of GRB 060206. "FE" and "RE" represent the forward shock emission and the reverse shock emission, respectively. The dotted line represents the contribution from Jet $A$. The thick, dashed line and the thin, dashed line correspond to the emissions from the forward shock and the reverse shock of Jet $B$, respectively. The dash-dotted lines represent the emission from Jet $B$ taking into account the zero-time effect. The thick, solid line includes the contribution from both Jet $A$ and Jet $B$. $R$-band data are taken from Monfardini et al. (2006), Stanek et al. (2007) and Woźniak et al. (2006).

\section{Summary and discussions}

We have presented a solution for the remarkable rebrightening observed in the afterglow of GRB 060206, which is attributed to emission from an off-axis beamed jet originating from the late activity of the central engine after the prompt gamma-ray emission phase. We have argued that the precession of the torus or accretion disk of the central engine has caused the two jets to move in different directions. Although we only attempt to describe the large rebrightening, it is reasonable to speculate that the five bumps in the $R$-band afterglow light curve of similar profiles may have the similar origins: emission from different delayed off-axis jets ejected by the GRB central engine. The difference between the temporal indices of the five post-bump light curves indicates that the electrons in different jets have different spectral indices $p$ for their electron distributions. As a result, the spectra exhibit an SED evolution, especially when the separate jets have comparable contributions to the total emission. This can be naturally explained by the significant SED evolution in GRB 060206 detected by the Liverpool Telescope (Monfardini et al. 2006).

The isotropic energy of the second jet is more than one order of magnitude higher than the first jet in our scenario. The collimation-corrected energy of Jet $B$ is $\sim 4.3$ times larger than Jet $A$, which is larger than the energy required to explain the big rebrightening in the energy injection model as mentioned in Sect. 1. The precise mechanism that triggers the central engine again remains unknown. One possibility is that a mass of debris falls back onto the central compact object, generating another more energetic jet. Since our scenario can reproduce well the observations of the large bump in GRB 060206, as a conservative extrapolation, we propose that GRB 970508 and GRB 060210 , which display remarkable rebrightening, may have in addition a precessing torus or accretion disk.

A further consequences of our scenario is that the jet break is determined by the off-axis second jet rather than the first one, which produces the main burst. In this case, we cannot measure the isotropic energy of the second jet directly and must fit the afterglow data to obtain an estimation. When a large bump appears in GRB afterglows, we are therefore unlikely to be able to derive the jet opening angle, using the break time and isotropic gammaray energy release, because these two quantities originate in two different jets (Stanek et al. 2007).

Finally, several models could be applied to explain afterglow light curves exhibiting rebrightening. These include variable external density profiles (Lazzati et al. 2002), refreshed shocks (Granot et al. 2003; Björnsson et al. 2004), and angular dependence of the energy profile on the jet structure (Nakar et al. 2003), each of which can play a role. Peculiar behavior in light curves caused by the precession of the central engine was discussed by Reynoso et al. (2006), although more observations are required to identify its true nature. 
Acknowledgements. We would like to thank the anonymous referee's constructive comments and suggestions which improved our paper significantly. X.W. Liu thanks D.M. Wei for his encouragement to accomplish this work. We thank Y.F. Huang, Y.W. Yu, Y. Li and L. Shao for helpful discussions. This work was supported by the National Natural Science Foundation of China (grants 10473023, 10503012, 10621303, and 10633040). XFW gratefully acknowledges Re'em Sari during his visit to Caltech, also thanks the supports of China Postdoctoral Science Foundation, K.C. Wong Education Foundation (Hong Kong), and Postdoctoral Research Award of Jiangsu Province.

\section{References}

Boyd, P., Hunsberger, S., \& Gronwall, C. 2006, GCN Circ., 4684 Björnsson, G., Gudmundsson, E. H., \& Jóhannesson, G. 2004, ApJ, 615, L77 Dai, Z. G., \& Lu, T. 2002, ApJ, 565, L87

Fan, Y. Z., Dai, Z. G., Huang, Y. F., \& Lu, T., et al. 2002, Chin. J. Astron. Astrophys., 2, 449

Freedman, D. L., \& Waxman, E. 2001, ApJ, 547, 922

Fynbo, J. P. U., Starling, R. L. C., Ledoux, C., et al. 2006, A\&A, 451, L47

Frail, D. A., Kulkarni, S. R., Sari, R., et al. 2001, ApJ, 562, L55

Galama, T. J., Groot, P. J., van Paradijs, J., et al. 1998, ApJ, 497, L13

Granot, J., Nakar, E., \& Piran, T. 2003, Nature, 426, 138

Huang, Y. F., Gou, L. J., Dai, Z. G., \& Lu, T. 2000, ApJ, 543, 90

Jin, Z. P., Yan, T., Fan, Y. Z., \& Wei, D. M. 2007, ApJ, 656, L57

Kobayashi, S. 2000, ApJ, 545, 807

Kobayashi, S., Zhang, B., Mészáros, P., \& Burrows, D. N. 2007, ApJ, 655, 391 Kumar, P., \& Piran, T. 2000, ApJ, 532, 286

Lazzati, D., Rossi, E., Covino, S., Ghisellini, G., \& Malesani, D. 2002, A\&A, 396, L5
Liang, E. W., Zhang, B., O’Brien, P. T., et al. 2006, ApJ, 646, 351 MacFadyen, A. I., \& Woosley, S. E. 1999, ApJ, 524, 262

Morris, D. C., Burrows, D., Gehrels, N., et al. 2006, GCN Circ., 4682

Monfardini, A., Kobayashi, S., Guidorzi, C., et al. 2006, ApJ, 648, 1125 Nakar, E. 2007, Phys. Rep., 442, 166

Nakar, E., \& Granot, J. 2007, MNRAS, 380, 1744

Nakar, E., \& Piran, T. 2004, MNRAS, 353, 647

Nakar, E., Piran, T., \& Granot, J. 2003, NewA, 8, 495

Nousek, J. A., Kouveliotou, C., Grupe, D., et al. 2006, ApJ, 642, 389

O'Brien, P. T., Willingale, R., Osborne, J., et al. 2006, ApJ, 647, 1213

Palmer, D., Barbier, L., Barthelmy, S., et al. 2006, GCN Circ., 4697

Rees, M. J., \& Mészáros, P. 1998, ApJ, 496, L1

Reynoso, M. M., Romero, G. E., \& Sampayo, O. A. 2006, A\&A, 454, 11

Rybicki, G. B., \& Lightman, A. P. 1979, Radiative Processes in Astrophysics (New York: Wiley)

Sari, R., \& Esin, A. A. 2001, ApJ, 548, 787

Sari, R., \& Mészáros, P. 2000, ApJ, 535, L33

Sari, R., \& Piran, T. 1995, ApJ, 455, L143

Sari, R., Piran, T., \& Narayan, R. 1998, ApJ, 497, L17

Stanek, K. Z., Dai, X., Prieto, J. L., et al. 2007, ApJ, 654, L21

Woźniak, P. R., Vestrand, W. T., Wren, J. A., et al. 2006, ApJ, 642, L99

Wijers, R. A. M. J., \& Galama, T. J. 1999, ApJ, 523, 177

Woosley, S. E. 1993, ApJ, 405, 273

Wu, X. F., Dai, Z. G., Huang, Y. F., \& Lu, T. 2003, MNRAS, 342, 1131

Yan, T., Wei, D. M., \& Fan, Y. Z. 2007, Chin. J. Astron. Astrophys, 7, 777

Zhang, B., \& Kobayashi, S. 2005, ApJ, 628, 315

Zhang, B., Fan, Y. Z., Dyks, J., et al. 2006, ApJ, 642, 354

Zou, Y. C., Wu, X. F., \& Dai, Z. G. 2005, MNRAS, 363, 93 